\title{
Dilemma and Transcendence: Self-Compassion in Epidemic Crisis
}

\author{
Zhao Kang ${ }^{1, *}$, Youli Kang ${ }^{2}$, Qingfeng Shi ${ }^{1}$
}

\begin{abstract}
${ }^{1}$ School of Education, Research Center for Mental Health Education, China West Normal University, Nanchong, Sichuan, China

${ }^{2}$ Psychological Counseling Center for University Students, China West Normal University, Nanchong, Sichuan, China ${ }^{*}$ Corresponding author. Email: kangzhao168@126.com
\end{abstract}

\begin{abstract}
Currently the rapid development of society has led to the increasement of pressure on individual survival and the prominence of the lack of self-compassion, which has resulted in the loss of individual role and the crisis of cultural identity. Therefore, it necessary to carefully analyze the current situation that individuals lack self-compassion in the epidemic crisis, deeply understand the practical meaning of self-compassion and its important value for individual growth, and advocate several paths such as strengthening the subject's self-identification, actively promoting self-improvement and creating a good atmosphere of humane care so as to cultivate self-compassion awareness and the healthy development of individuals.
\end{abstract}

Keywords: Epidemic crisis, Self-compassion, Self-actualization.

\section{困境与超越: 疫情危机下的自我关怀}

康钊 ${ }^{1, *}$, 康有黎 ${ }^{2}$, 石庆丰 ${ }^{1}$

\footnotetext{
1 西华师范大学教育学院, 心理健康教育研究中心, 南充, 四川, 中国

2 西华师范大学大学生心理咨询中心, 南充, 四川, 中国

*通讯作者. 邮箱: kangzhao168@126.com
}

\section{中文摘要}

当前社会极速发展导致个体生存压力不断增加，自我关怀缺失问题凸显，并由此引发个体身份的迷失和文化认 同危机。认真分析疫情危机下个体自我关怀的缺失现状，深刻体认自我关怀的现实意蕴及其对个体成长的重要 价值，主张通过强化主体自我认同、积极促进自我提升与营造良好人文关怀氛围等路径培育个体的自我关怀意 识, 助推个体健康发展。

关键词: 疫情危机, 自我关怀，自我实现

\section{1. 引言}

2020年初爆发的新冠病毒肺炎疫情及其衍生出来 的诸多危机事件, 给我们当前的各项管理工作提出许 多新的挑战, 特别是当前社会极速发展导致个体生存 压力不断增加, 让我们不得不正视当前个体自我关怀
缺失这一现实问题。全力做好疫情危机的科学应对工 作，深刻体认自我关怀的现实意蕴及其对个体成长的 重要作用, 积极探索疫情危机下自我关怀教育的新思 路，全面提升广大人民群众的心理健康水平，成为时 代的紧急呼唤。 


\section{2. 疫情下个体自我关怀缺失现象透视}

\section{1. 自我隔离与封闭}

新型冠状病毒肺炎疫情来势凶猛, 由于病毒传染 性极强, 加上口罩等防护物质紧缺、确诊病例数字惊 人、封城禁行等各种信息纷至沓来, 带给人们更多的 是恐惧、焦虑甚至愤怒; 逆行出征、寒风送别、脸上 勒痕、倒地睡姿等动情画面广泛传播, 感动、担心甚 至心疼之情油然而生; 居家隔离、物质抢购、禁止聚 集等带给大家些许伤感、无助甚至绝望。随着个体居 家时间越来越长，以及希望疫情早日结束的心理预期， 个体对其自身生活的掌控感似乎越来越弱, 从疫情突 发初期的应激状态慢慢转入较为持久的焦虑与紧张 状态。各种复杂情感不断交织, 严重撕裂个体的内心 平衡, 部分民众甚至于因此而抓狂, 极度的焦虑与恐 慌心理造成个体自我封闭而与外界完全隔离, 将自己 置身于真空地带, 既对他人漠不关心, 对自己也生无 可恋，严重影响个体的正常生活状态。

\section{2. 铋无忌惮的自我放纵}

突如其来的疫情打乱多人的原初计划, 个体安逸 舒适的心情随着疫情的扩散慢慢变得紧张焦虑, 更害 怕自己和身边的人受到病毒的侵害。人们关注的焦点 也从娱乐头条、贺岁档片以及体育赛事等转移到疫情 消息上面。面对疫情现实, 许多人无所适从, 要么贪 恋被窝, 要么暴饮暴食, 要么沉溺网络, 肆无忌惮的 无序生活态势让自己变得更加邋昀与懒惰。许多人把 较长的疫情期变成自己懒惰与放纵的借口, 因为謷夜 打游戏, 或者聚众奢博等等原因而诱发的极端事件并 不少见。疫情期间个体工作的“不上班”或者“居家上 班”模式，让部分人找到“放飞自我”的机会。特别是 那些不能抵御外界诱惑、约束自我欲望而缺乏自律的 个体, 犹如脱缰的野马, “想干啥就干啥”, 自我关怀 演变成极度的自我放纵。同时, 疫情期间许多个体的 权利意识不断高涨, 但规则意识与公共精神却严重滞 后, 我行我素, 必然造成各种悲剧的出现。表现看似 自我关怀, 实则给家庭带来诸多烦恼与痛苦; 看似痛 快过瘾, 实则严重损害自己的身体健康。

\section{3. 过度地苛责自己}

疫情防控时期不少个体特别是身处疫区的民众因 疫情扩散导致对家人关心不足而怨恨自己, 部分参与 救助的医护人员同样因为自己无力回天而深深自责。 正因为这样, 有时连对自身加以关怀可能都会显得十 分困难。一方面在面对疫情时自己是那么的无助, 另 一方面则更有可能会为了成全他人放弃自己的合理 需求。这种极度地要求自我、责备自我势必造成对个 体自身的极大压力, 甚至可能变得十分危险。心理学 研究明确指出: 自我关怀的能力和对他人关怀的能力 两者之间并无太大关联。在当前社会中, 无私奉献、 乐于助人的好人好事层出不穷, 许多人总是喜欢把所
有的美好，所有的宽容，所有的善意都施加给他人， 但对自己却缺乏基本的关怀，这正是个体自我关怀能 力较弱而导致的结果。大多数自我苛责的念头其实像 是一种个体自身的防御性行为, 可促使我们做好各项 工作，但过度苛责会让自己陷入深深的悔恨与兼疚中 而难于自拔，极不利于个体的身心健康。

\section{4. 极度的自我中心}

在疫情防控非常时期, 明知防控疫情的最好方式 就是宅在家里, 有些人不愿意戴口罩却四处游荡, 这 样可能增加自身和他人的感染风险; 部分游客违反疫 情期间的相关规定，躲避地方医护人员，拒不接受隔 离观察, 或在国内外肆无忌惮地到处乱窝, 无视所在 国家法律法规，既饱受诟病与耻笑，还可能闯下大祸， 害人害己。我们的身边也不乏这类人, 那就是每当出 现令他们不舒心的事情时，他们总是表现出极度的自 我关怀。这类人总是无法客观理性地看待自己所处的 状况是否艰难, 实际上就是典型的自我中心意识在作 怪。还有一部分人大量了解疫情信息, 甚至于完全将 自己浸泡在疫情消息的海洋中, 不懂篮选与过滤, 容 易在得知他人的创伤，如看见一线医生或患者离世等， 好像自己曾经亲临创伤现场一般, 内心承受着莫名的 巨大痛苦, 甚至产生绝望意识。这种负性情绪如果长 时间无法得到排解，则极易成为替代性创伤的新宿主。 当然, 这可能也是部分人身上存在某种内在需要, 想 要通过替代性创伤来获取某种并存感, 通过共同处于 被创伤的位置来获取些许安全感, 但本质上同样无法 摆脱自我中心的桎梏。

\section{3. 个体自我关怀的现代意蕴与价值呈现}

关怀意即关心、帮助、爱护与照顾之意，是一种 个体身上表现出来的发自内心的善意表现。传统意义 上的关怀多指上对下或集体对个人的关心，现代研究 不断拓展其理论蕴含, 认为关怀是积极主动的, 而非 消极被动的，不管是对他人还是对自己。由此将关怀 区分成关怀他人与自我关怀两种不同的形式。自我关 怀就是自己对自己的关心，是个体对自身的一种关切 和设计。美国教育心理学家Kristin Neff (2003) 指出, 自我关怀是一个人有能力认识到自己正在经历某种 令人压抑痛苦的体验, 同时又有能力去感知这种体验 所带来的感受, 并且还有能力在这个过程中用足够的 爱与善意来照料自己, 这种照料的方式可以是精神上 的、情感上的、身体上的，也可以是某种特定的行为 [1]。朱伟 (2009) 主张从哲学和伦理学的角度来审视 自我关怀, 认为自我关怀是指个体对自身德性伦理的 积极关注, 关心个体的自我发展和自我完善[2]。盖岩 （2007）通过对法国思想家福柯（M.Foucault）生存 美学中相关要素的分析, 提出自我关怀是一种自我锻 造的生活艺术，其本质在于建构一个充分自由的自身， 真正地实现对于个体自身的关怀，抛弃传统道德规范 的约束，使个体在同时考虑他人快乐的同时亦能实现 自身的幸福快乐。他认为个体的自我关怀包含四大基 
本要素, 即认识自身、反思自身、精神修炼与拯救自 身[3]。德国克里斯汀娜·布莱勒(2017)认为自我关怀是 爱和勇气的源泉, 是让生命强大和我们对抗人生所有 苦难的唯一方式。由此，自我关怀就是指个体对自身 的关爱、关注与关心，包括个体对其自身作为社会生 活主体的关爱、对自己所从事的职业的关注以及对自 我发展和自我完善的关心[4]。

综合当前对于自我关怀的相关研究，自我关怀者 应当具备三个重要特征: 第一, 善待自己。善待自己 是指个体要以和善友爱的方式理解和认同自己, 学会 接纳自己的瑕疵乃至失败, 而不是一味地严厉批评和 苛责自己。从一定程度上说, 善待自己意味着个体能 够清楚觉知对自我的无情伤害, 并有效促成内心交战 的终止。第二, 共通人性。所谓共通人性是指个体能 够充分体悟到人我之间在生命体验方面的心灵感应 与高度契合, 而不是简单地被自己曾经经历的痛苦加 以孤立或隔离。同时, 个体能够充分体认人类生命共 同体的客观存在且与自身成长息息相关, 真正学会自 我接纳而非自我怜悯。我们在理解共通人性时, 需要 充分意识到无助感和失落感在每个个体身上都可能 出现。自我关怀并不要求人们忘记自身痛苦的遭遇, 而是充分理解自己在困难时刻所体验到的痛苦正如 他人在困难时刻所体验到的痛苦。第三，静观当下。 静观当下要求对我们自身的体验持以平衡的觉察, 而 不是随性忽视或无限夸大我们的痛苦经历。静观意即 对此时此刻发生的事情能够保持足够的清醒和非评 判性的接纳, 也就是我们平常所说的直面现实与活在 当下。我们需要学会客观理性地看待各种危机, 才能 对当前的不良境遇抱以最大的和有效的关怀。有学者 将静观称为“元察觉”, 即察觉到察觉。它能够让我们 学会清晰地认知自己的处境, 并对困难时局做出更加 有效的反应。

众多研究表明, 危机状态下个体自我关怀的缺失 极易诱发个体身份的迷失和文化认同危机。我们强调 主体的自我关怀, 其实质在于有效促进个体身心、情 感、精神等方面达成高度和谐与平衡的状态 [5]。事实 上, 个体在自我提升与自我反思的同时, 也能增强主 体自身对他人的关怀意识。因此, 个体的自我关怀具 有促进自身健康和关怀他人两大功能。在新冠疫情尚 未完全解除的时间里, 我们更应当重视对自身进行积 极的自我关怀。首先, 自我关怀可以增强个体自身的 情绪修复能力而使情感弹性更强, 增强自身对新冠病 毒的身体免疫能力, 同时还可以改善人际关系, 学会 更多地关心和理解他人, 提高个体对现实生活的整体 满意度, 自然也有助于降低因疫情而导致的焦虑与抑 郁, 以及减轻居家隔离或工作需要而带来的生活压力 和创伤影响。其次, 自我关怀能够提升个体的行为动 机, 促进个体以积极的姿态面对现实并促进自我不断 完善。学会自我关怀的个体往往能够严格要求自己, 对自身的健康与快乐充满深深的渴望与期待, 能够积 极付诸努力去尝试做好那些利于自己学习和成长的 事情。今天，我们特别推崇重视个体人文精神的培养
促进个体人格的健全发展, 其实本质上就是主张实现 一种普遍的人类自我关怀，即对个体人格的高度尊重、 人生价值的充分肯定与命运的积极关怀等。

\section{4. 疫情危机下自我关怀的实现路径探讨}

\section{1. 理性认识疫情特征，创设人文关怀氛围}

我们对疫情应对工作要有科学理性的认识, 病毒 的传染性较强, 初期不少参与一线救治的医护人员也 曾被感染，所以我们必须加强日常防范，阻断疫情病 毒可能的传播途径，有效遏制病毒扩散。目前虽然尚 未研制出相应的高效疫苗和特效防治药物, 但国家相 关部门正在努力推进这项工作的进程。因此, 我们要 接纳当前的现实状况, 积极响应党和国家号召, 全力 作好疫情期间的自我防范工作。要警惕和避免消极的 应对方式，如消极逃避、自我封闭或对各种消息不加 辨识, 因为这些都可能加剧自己的负面情绪体验。同 时，应当重视创设充满人际关怀的和谐氛围，积极关 注社会弱势群体，做好对他们的经济救助与精神培育 工作，保证弱势群体在疫情非常时期的基本利益。积 极引导个体在参与疫情防控时期社会公共生活的过 程中形成基本的社会公德意识与现代公民精神，自觉 遵守社会公共秩序, 关心社会公共事务, 帮助个体深 刻体认权利和义务的高度统一性，既不放弃自己应当 享有的各种权利，同样不能对侵害自己合法权益的事 情置之不顾，更不能滥用自己的权利去胡作非为。

\section{2. 不求全责备, 学会爱护与善待自己}

自我关怀的个体必定承认自己也有七情六欲, 我 们也可能遭遇困难挫折, 特别是当我们确实需要他人 的帮助时, 要及时主动地向他人敞开胸怀。德国克里 斯汀娜·布莱勒博士明确指出个体要学会接纳自己的 不完美, 因为那是我们完整的一部分。自我关怀的行 为经常被忽视, 而事实上爱自己可能比其他所有行为 都更为重要。如果我们能够给予自己足够的关怀与爱 护，以更具弹性的方式去应对所有的不确定性，包括 前进道路上的挫折和困难，一切都可能变得更加美好。 我们要深深地意识到, 我们不是孤军奋战, 我们有同 事、朋友、团队，有社会主义和谐大家庭，学会向他 人和社会寻求帮助，共同度过疫情难关。当我们自己 遭遇困难、挫折或失败而感到极度痛苦甚至讨厌自己 时, 要像我们对待自己要好的朋友或亲人那样, 能够 带着善意与耐心帮助照顾好当下那个无助的自己度 过难关。我们要学会用自我关怀来调节自身当下体验 到的压力与不适状况，及时化解自己身上的诸如恐惧、 悲伤、绝望或愤怒等负面情绪。因为关怀总是“我”在 关怀，如果“我”对自己生命体的存在都没有关怀意识， 那么很难想象 “我”对关怀他人或他物具有强烈的意 识。自我关怀就是要让个体自己学会正确地认识自我 与悦纳自我, 学会热爱生活与享受现实生活, 提升主 体自身的生活幸福指数。 


\section{3. 学会知足常乐, 学会认同与包容他人}

人生一辈子可以追求的东西的确太多太多，但是 我们却无法做到事事都能心随己愿，很多事情我们穷 其一生的时间去追求, 到头来却也可能一无所获。所 以, 从另外一个角度来说, 我们要学会欣赏自己通过 努力所获取和拥有的一切财富, 学会随遇而安与知足 常乐, 适度的自我满足同样是一种人生智慧。同时, 我们要学会认同接纳和包容他人。真正的自我关怀不 是年少时的无敌与轻狂, 不是初涉人事的单纯与肤浅, 更不是无知无畏的冲动与浮躁, 而是要深刻体认包容 和认同。同时, 当你关心别人的时候, 通过关怀自己 向对方展示自我关怀的重要性, 这是很有帮助的, 而 且还能阻止对方对他自己的忽视。事实上, 个体只有 生活在复杂的社会关系网络之中才能真正感知自我 存在并充分体悟自我价值, 通过在复杂的社会网络中 建立良好的人际关系, 促成个体不断地提升自我和实 现自我。

\section{4. 学会自强自立, 保持有界限的自我}

突如其来的较长假期, 是我们对自己人生的态度 和亲情维护的一种考验, 所以不能把疫情放假变成自 己懒惰与放纵的借口, 而应当重视不断提升自己的能 力与人生价值。真正的自我关怀不是自我的完全封闭, 也不是消极应对和对外界的极度依赖, 更不是自我菲 薄与自私膨胀, 而是要学会自强与自立, 将自己科学 融入真实的现实社会之中。特别是当我们自己身处绝 境时, 经历过风吹雨打和痛苦磨砺之后, 才更能让我 们保持内心的澄明。要充分认识现实社会就是一个人 生大舞台, 社会上的人形色各异, 不能按自己的标准 去强求每一个人, 并非每个人都乐善好施。我们要理 解别人, 别人没有帮助你也许有别人的苦哀; 我们要 学会原谅与宽恕, 宽恕别人的错误, 宽恕别人就是宽 恕自己, 更不能拿别人的错误来惩罚自己。有时候, 我们可以尝试暂时停下手中的工作, 用你自己的身体 去认真体会下可能会感到的任何压力、痛苦、怀疑、 愤怒、沮丧与焦虑等负性情绪, 让自己充分明白自己 感受到这些情绪是正常的。同时, 我们要积极促成自 己在追求真善美的过程中自觉遵守社会道德准则, 不 断获得对自己个性的充分发展和完美德性的塑造。拥 有德性人格的个体能够在满足社会幸福的前提下充 分关注自身的幸福指数, 能够自己真正实现关怀自己， 并为实现个体与他人幸福而不断努力。

\section{5. 积极进行自我暗示, 摆脱不良心理困扰}

置身于当前的疫情环境, 我们应当清楚地意识到 关注我们自身的需要可以提升主体的幸福感, 生活中 忽视自我关怀则可能导致个体的焦虑情绪增加、注意 力无法集中、容易生气动怒或倍感身心疲㤂。同时, 个体还可能遭受诸如睡眠质量不高、人际关系不睦、 自尊心与共情能力减弱等不良心境困扰。持续的疫情 压力状态导致个体缺少适度的自我关怀, 甚至可能会
有罹患抑郁症和心脏病等疾病的风险。因此, 我们应 当重视以积极的态度面对现实生活, 采取积极的自我 暗示方法, 提升自己的内在信念, 如坚信自己没被感 染自己是十分幸运的, 自己现在衣食无忧自己是幸福 的, 自己是有能力挺过难关的, 居家隔离期间自己同 样是有责任的, 等等。也就是说, 主体自身的紧张、 焦虑与无助感其实大家同样也都能感受到, 正因为别 人和自己是一样的, 所以我们自己其实并不孤独, 生 活中的“你”“我”“他”都不可缺少。让疫情环境下的个 体清晰地觉察自己的当前处境, 接纳自己的当下生活, 学会自我关怀, 放松自我身心, 管理好自己的情绪, 充分享受美好幸福的人生。

\section{致谢}

本文为西华师范大学基本科研业务费项目《西部 地区乡村振兴的文化铸魂研究: 中国传统文化与西方 心理学整合视角》（19B003）; 南充市社科研究“十 三五”规划2020年度项目： 《乡村振兴过程中文化铸 魂的现代意蕴与路径研究》（NC2020B101）的阶段 性成果之一。

\section{REFERENCES}

[1] Neff, K.D.Self-compassion: An alternative conceptualization of a healthy attitude toward oneself. Self and Identity, Vol.2, No.2, 2003, pp.85-102.

[2] Zhu Wei. Self-compassion: the path of teacher self-development. Educational research and experimentation, Vol.5, 2009, pp.4-6.

[3] Gai Yan. Self-compassion, a Living Art of Self-Forge -- A Brief Analysis of Several Elements in Foucault's Survival Aesthetics, Social Science Forum, Vol.10, 2007, pp.21-22.

[4] Christina Blahler, translated by Liu Xiao. Self-compassion: the only way to live a strong life. Beijing: Beijing United Publishing Company, 2017.

[5] Burkhardt MA, Nagai-Jacobson MG. Nurturing and caring for self. The Nursing Clinics of North America, Vol. 12, No.1, 2001, pp.23-31. 